\title{
Cardiovascular risk factors in Australia: trends in socioeconomic inequalities
}

\author{
Stan Bennett
}

\begin{abstract}
Study objective-To examine trends in socioeconomic inequalities in cardiovascular risk factors using educational attainment to indicate socioeconomic status. Design - Behavioural data, physical measurements, blood pressure, and lipid determination collected in three, successive multicentre cross sectional community surveys conducted in 1980,1983 , and 1989. Setting - The six state capital cities of Australia; Sydney, Melbourne, Brisbane, Adelaide, Perth, and Hobart.
\end{abstract}

Participants - A total of 19315 randomly selected respondents stratified by age (2544, 45-64) and sex.

Results-During the 1980s, average blood pressure declined for each level of educational attainment. Dietary messages to reduce the intake of saturated fat had little effect on the lipid profile of any population group. Height and educational attainment were positively associated. Women increased in weight from between 2 to $4 \mathrm{~kg}$ depending on age and educational attainment while older men experienced increases of around $2.5 \mathrm{~kg}$ regardless of ecucational attainment. Advice to avoid salt was adopted across the spectrum of educational attainment but with no suggestion that the socioeconomic gradient, which favoured the more highly educated, was diminishing. Men of all education levels responded positively to the antismoking initiatives of the 1980 s but the relative disadvantage of those of lower education was maintained. Among women, the decline in smoking was less among those in the low education group. The prevalence of moderate to heavy drinkers was higher in men of lower educational attainment but declined significantly over the period. Walking for recreation or exercise became more popular, especially among older men of low education, while the prevalence of aerobic exercise and vigorous exercise remained largely unchanged. Overall, the clear socioeconomic gradient between leisure time physical activity and education attainment remained.

Conclusions-The lower socioeconomic group has improved its risk factor profile but its relative disadvantage compared with the higher socioeconomic group persists. Health promotion activities in Australia seem to have been effective in reaching the lower socioeconomic groups but the challenge to reduce inequalities remains. The steady increase in educational attainment in Australia may have been an important factor in the general improvement in the nation's risk factor profile and in the decrease in mortality from coronary heart disease.

\section{( $f$ Epidemiol Community Health 1995;49:363-372)}

In Australia, as in other developed countries, people of lower socioeconomic status are presently at greater risk of cardiovascular disease. This relationship has been documented for mortality from coronary heart disease and stroke, in men and women, and for morbidity and risk factors. ${ }^{1-10}$ There is evidence for men that the appreciable decline in cardiovascular mortality observed in Australia since the late 1960s was initially greater among the higher socioeconomic groups: that is, that socioeconomic inequalities were widening. ${ }^{2-411}$ This phenomenon has also been observed in Britain and the United States. ${ }^{1213}$

Within developed countries, the pattern of higher rates of illness and death among people of lower socioeconomic status holds for most diseases. ${ }^{1415}$ Reasons for this association are unclear and potential explanations include external factors and personal characteristics and behaviours of the individual. These include living and working conditions; access to health care services; cultural influences; aspects of social support; social mobility related to health; knowledge, attitudes, and values; and behavioural risk factors. ${ }^{1016-18}$

Many risk factors, biomedical and behavioural, are strongly related to socioeconomic status and their unequal distribution in society is known to contribute to the inverse gradient between socioeconomic status and cardiovascular mortality. ${ }^{1920}$ It follows that monitoring socioeconomic differentials in cardiovascular risk factors is important for understanding trends in the socioeconomic distribution of cardiovascular disease, and for supporting the development of cost effective and equitable strategies for its prevention and treatment.

In recent years, a wide variety of initiatives has been undertaken to improve the risk factor profile of Australians, and favourable trends were observed in the prevalence of cigarette smoking, some dietary behaviours, and participation in lighter exercise among adults. ${ }^{21}$ Mean blood pressure declined significantly but changes in blood lipids were small. Body fatness increased noticeably during the past decade, especially in women. What is not known is 
whether socioeconomic groups responded differently in adopting healthier lifestyles and how this affected socioeconomic inequalities in biomedical risk factors. To answer these questions, data from three successive risk factor surveys conducted in Australia during the 1980s were examined for socioeconomic inequalities in behavioural and biomedical risk factor levels and for trends in any such inequalities over time.

Measures of education, occupation, and income, either singly or as a composite score, are commonly used as indicators of socioeconomic status. These indicators are invariably correlated but are believed to measure different aspects of socioeconomic circumstances. ${ }^{2223}$ Only educational attainment was collected in a consistent manner across all three surveys and it has therefore been used to indicate socioeconomic status in this analysis. Educational attainment is considered to be related to values and attitudes which result in healthier behaviours, greater use of preventive health services, and a more positive social and physical environment. ${ }^{24}$ It is usually complete by early adulthood and is available for all men and women regardless of employment status. ${ }^{24}$ Education has been found to be strongly associated with cardiovascular mortality ${ }^{25}$ and with coronary risk factors. ${ }^{2627}$ It has been recommended as the most judicious single measure of socioeconomic status for use in epidemiological studies. ${ }^{22}$

The purpose of the analysis was to detect any associations between educational attainment and levels of risk factors, to examine their consistency across age and sex groups, and their replication over time.

\section{Methods}

STUDY DESIGN

Data for this analysis have been provided by the National Heart Foundation's risk factor prevalence study, comprising multicentre surveys conducted during 1980, 1983, and 1989. These surveys were not longitudinal but were designed to provide cross sectional estimates for the Australian adult urban population at intervals. The three surveys used basically the same methods. ${ }^{28}$ A systematic probability sample of adults was selected from federal electoral rolls for each capital city in Australia. Over $60 \%$ of Australians live in these capital cities and voter registration is compulsory for all Australian citizens aged 18 years and older Adults living in other urban or rural areas were not covered by the study. Those identified were invited by post to attend a local survey centre at a specific appointment time for a free check of risk factors for heart disease. Each survey achieved a response rate close to $75 \%$. At the centre, participants completed a self administered questionnaire and were then referred to a nursing sister for physical and blood pressure measurement and blood sampling. Staff were trained in standardised procedures and measuring techniques, and data quality was monitored.
EDUCATIONAL ATTAINMENT

Educational attainment was determined by the response to the request, "please indicate the highest level of education you have completed". Education was categorised into groups labelled low (no schooling, primary school, or some high school), medium (completed high school at year 12 or equivalent), and high attainment (completed tertiary qualification at university, college of advanced education, or other tertiary institution).

\section{BIOMEDICAL RISK FACTORS}

Systolic (SBP) and diastolic (DBP) blood pressures were determined as the mean of two consecutive readings taken five minutes apart from the right arm, with the participants seated, and using normal mercury sphygmomanometers. Hypertension was defined as $\mathrm{SBP} \geq 160 \mathrm{mmHg}$ and/or $\mathrm{DBP} \geq 95 \mathrm{mmHg}$ and/or taking tablets for blood pressure.

Morning fasted blood specimens were analysed for plasma total cholesterol (TC), highdensity lipoprotein (HDL) and triglycerides (TG). Low density lipoprotein (LDL) was calculated as TC-HDL-TG/2.19 if TG $<4.5 \mathrm{mmol} / 1{ }^{29}$ Trends were also examined for the ratio of TC to HDL. High TC was defined as $6.5 \mathrm{mmol} / \mathrm{l}$ or greater. Low HDL was defined as less than $0.91 \mathrm{mmol} / 1$ for men $(35 \mathrm{mg} / \mathrm{dl})$ and less than $1.16 \mathrm{mmol} / 1(45 \mathrm{mg} / \mathrm{dl})$ for women. ${ }^{30}$

Height was measured to the nearest $\mathrm{cm}$, weight to the nearest $0.1 \mathrm{~kg}$ ( $1 \mathrm{~kg}$ in 1980). Body mass index (BMI) was calculated as weight (in $\mathrm{kg}$ ) divided by the square of height $\left(\mathrm{m}^{2}\right)$ after deducting $1 \mathrm{~kg}$ from the measured weight as an allowance for weight of clothing. For both sexes, overweight was defined a $25<\mathrm{BMI} \leq 30$; and obese as $\mathrm{BMI}>30$.

\section{BEHAVIOURAL RISK FACTORS}

The questionnaire asked, "Do you add salt to your food after it is cooked?" (1983 and 1989) and, "How often do you eat the fat on meat?" (1980 and 1989). For both questions this analysis used the response, "rarely or never" as an indicator of reduced risk.

Smoking status was determined from self reported questionnaire data. Current smokers were defined as smoking $\geq 1$ manufactured cigarettes a day, $\geq 1 \mathrm{~g}$ "hand-rolled" cigarette tobacco per week, $\geq 1$ cigars per week, or $\geq 1 \mathrm{~g}$ pipe tobacco per week.

Alcohol intake was classified as never or occasional ( $<1$ drink per week), light (1-27 drinks per week for men, 1-13 drinks per week for women) or moderate to heavy $(\geq 28$ drinks per week for men, $\geq 14$ drinks per week for women), consistent with the National Health and Medical Research Council recommendations for "responsible drinking behaviour". ${ }^{31}$

In the 1983 and 1989 surveys, respondents were asked about exercise taken for recreation, sport, or health fitness purposes in the previous two weeks. This included vigorous exercise (defined as causing breathlessness, puffing and panting), less vigorous exercise, and walking. 
Vigorous exercise for three or more times a week and an average of 20 minutes or more per session was classified as aerobic exercise. No leisure time exercise of any kind was also used as an indicator of cardiovascular risk. No account was taken of exercise at work.

MULTIPLE RISK FACTORS

The prevalence of individuals with two or more of the three classic risk factors for coronary disease was analysed for socioeconomic gradients and trends. The risk factors were defined as follows: high DBP ( $\geq 95 \mathrm{mmHg}$ ), high TC $(\geq 6.5 \mathrm{mmol} / \mathrm{l}$ ) and regular cigarette smoking (any number daily). All respondents were included whether or not they had fasted.

The prevalence of two or more biomedical risk factors (high DBP, high TC, low HDL, obesity) was also analysed as was the prevalence of two or more behavioural risk factors (regular cigarette smoking, no leisure time physical activity, moderate to heavy alcohol intake, almost always add salt to food).

\section{STATISTICAL ANALYSIS}

The trend analysis was based upon those variables common to the 1989 survey and the 1983 and/or 1980 surveys. The common age range was 25-64 years, and the centres common to each survey were in Sydney (two centres), Melbourne, Brisbane, Adelaide, Perth, and Hobart. On this basis, survey respondents numbered 5603 in 1980, 7615 in 1983, and 6097 in 1989.

All analyses were conducted separately for men and women stratified by age (25-44, 45-64 years) using $S A S$ software package, version 6. Age, as at 30 June of the survey year, was calculated from date of birth provided by the respondent.

For continuous variables (for example, blood pressure), associations with educational attainment over time were examined by analysis of covariance. The model included survey year, age, survey centre, and birthplace (Australianborn or overseas-born) as independent variables to allow for differences in the demographic composition of participants over time. An interaction term for educational attainment and year of survey was included to allow for different trends in different education levels. Age was included as a covariate because mean age varied between educational attainment groups even within the broad age strata considered. Use of oral contraceptives was introduced as a factor when analysing data on blood lipids for women aged 25-44.
Multiple logistic regression was used to examine changes in categorical variables over time (for example, proportion of smokers) using the same independent variables as for continuous variables. Using high education in 1980 as the reference group, prevalence odds ratios (ORs) and their $95 \%$ confidence limits (CI) (available from the author) were derived from the maximum likelihood parameter estimates of the model. ORs for selected risk factors were displayed graphically using a $\log _{2}$ scale to enable comparison of time trends above and below unity.

Certain data were excluded from the analyses. Data from non-fasted respondents and four extreme triglyceride values ( $\geq 30 \mathrm{mmol} / \mathrm{l}$ ) were excluded from blood lipid analyses. Data for pregnant women were excluded from all analyses which included BMI. Procedures for measuring height in Adelaide in 1980 deviated from the study protocol and these data (and BMI) were excluded from the analysis (weight measurements for Adelaide were included).

Missing data were negligible $(<0 \cdot 1 \%)$ for educational attainment, all survey design variables, and risk factors with the exception of BMI $(0.4 \%)$, TC (1.4\%), HDL (3.3\%), and TG (1.4\%). Information was maximised and records containing variables with missing data were only excluded from analyses in which those variables occurred.

\section{Results}

Post-secondary (high) education was reported more often by men and women, and by younger than older people (table 1). Over the study period there was an increase in the proportion who reported post-secondary education.

Table 2 shows time trends for each level of educational attainment, adjusted for age and survey design parameters. Table 3 shows differences between high and low educational attainment (inequalities) for each survey year, again adjusted for age and survey design parameters. Tables showing age-sex specific risk factor levels at each survey time, for each category of educational attainment are available from the author.

Generally, falls in blood pressure occurred at each level of educational attainment with consequent reductions in the prevalence of hypertension (table 2, fig 1 ). A socioeconomic gradient tended to favour those with higher educational attainment, however the gradient was not apparent at all points in time (table 3).

Socioeconomic gradients and time trends for TC and raised cholesterol were not pronounced (fig 1). Educational attainment and HDL were

Table 1 Sample distribution in relation to educational attainment, sex, age group, and year

\begin{tabular}{|c|c|c|c|c|c|c|c|c|c|c|c|c|}
\hline \multirow{2}{*}{$\begin{array}{l}\text { Education } \\
\text { level }\end{array}$} & \multicolumn{3}{|c|}{ Men 25-44y } & \multicolumn{3}{|c|}{ Men 45-64y } & \multicolumn{3}{|c|}{ Women 25-44y } & \multicolumn{3}{|c|}{ Women 45-64y } \\
\hline & 1980 & 1983 & 1989 & 1980 & 1983 & 1989 & 1980 & 1983 & 1989 & 1980 & 1983 & 1989 \\
\hline $\begin{array}{l}\text { Low (\%) } \\
\text { Medium (\%) } \\
\text { High (\%) }\end{array}$ & $\begin{array}{l}31 \cdot 6 \\
37 \cdot 0 \\
31 \cdot 4\end{array}$ & $\begin{array}{l}27 \cdot 3 \\
36 \cdot 4 \\
36 \cdot 3\end{array}$ & $\begin{array}{l}31 \cdot 8 \\
30 \cdot 5 \\
37 \cdot 7\end{array}$ & $\begin{array}{l}59 \cdot 3 \\
24 \cdot 2 \\
16 \cdot 5\end{array}$ & $\begin{array}{l}53 \cdot 5 \\
25 \cdot 9 \\
20 \cdot 7\end{array}$ & $\begin{array}{l}52 \cdot 1 \\
23 \cdot 2 \\
24 \cdot 7\end{array}$ & $\begin{array}{l}47 \cdot 3 \\
38 \cdot 3 \\
23 \cdot 7\end{array}$ & $\begin{array}{l}34 \cdot 9 \\
40 \cdot 4 \\
24 \cdot 8\end{array}$ & $\begin{array}{l}37 \cdot 2 \\
30 \cdot 1 \\
32 \cdot 7\end{array}$ & $\begin{array}{l}64 \cdot 8 \\
24 \cdot 6 \\
10 \cdot 6\end{array}$ & $\begin{array}{l}57 \cdot 3 \\
31 \cdot 9 \\
10 \cdot 8\end{array}$ & $\begin{array}{l}61 \cdot 7 \\
23 \cdot 1 \\
15 \cdot 2\end{array}$ \\
\hline No & 1437 & 2036 & 1647 & 1328 & 1704 & 1338 & 1462 & 2105 & 1740 & 1376 & 1769 & 1372 \\
\hline
\end{tabular}


Table 2 Trends ${ }^{\mathrm{a}}$ in risk factors in relation to educational attainment, sex, and age group

\begin{tabular}{|c|c|c|c|c|c|c|c|c|c|c|c|c|}
\hline \multirow[b]{2}{*}{ Variable } & \multicolumn{3}{|c|}{ Men 25-44y } & \multicolumn{3}{|c|}{ Men 45-64y } & \multicolumn{3}{|c|}{ Women 25-44y } & \multicolumn{3}{|c|}{ Women 45-64y } \\
\hline & Low & Med & High & Low & Med & High & Low & Med & High & Low & Med & High \\
\hline \multicolumn{13}{|l|}{ Biomedical risk factors } \\
\hline Systolic blood pressure $(\mathrm{mmHg})$ & $-4 \cdot 4 \ddagger$ & $-4 \cdot 6 \ddagger$ & $-1 \cdot 8^{*}$ & $-3.9 \ddagger$ & $-6 \cdot 1 \ddagger$ & $-3 \cdot 5^{*}$ & $-2 \cdot 9 \ddagger$ & $-2 \cdot 7 \ddagger$ & $-2 \cdot 6 \dagger$ & $-4.9 \ddagger$ & $-2 \cdot 8$ & $-5 \cdot 7 \dagger$ \\
\hline Diastolic blood pressure (mmHg) & $-3 \cdot 5 \ddagger$ & $-3 \cdot 7 \ddagger$ & $-2 \cdot 1 \ddagger$ & $-3.0 \ddagger$ & $-3 \cdot 8 \ddagger$ & $-3 \cdot 2 \ddagger$ & $-2 \cdot 0 \ddagger$ & $-1.5^{*}$ & $-2 \cdot 0 t$ & $-4 \cdot 1 \ddagger$ & $-4 \cdot 1 \ddagger$ & $-3.0 t$ \\
\hline Hypertension & $0.48 \ddagger$ & $0.43 \ddagger$ & $0 \cdot 72$ & 0.81 & $0.65 *$ & $0.44 \ddagger$ & $0.61^{*}$ & $0.43 t$ & $0.37^{*}$ & $0.64 \ddagger$ & 0.71 & $0.48 t$ \\
\hline Total cholesterol (TC) $(\mathrm{mmol} / \mathrm{l})$ & .06 & $-\cdot 13$ & $-\cdot 11$ & .01 & -.08 & -.09 & -.04 & .01 & .07 & $-\cdot 19 \neq$ & -.11 & -.15 \\
\hline Raised total cholesterol & $1 \cdot 23$ & $1 \cdot 04$ & 0.71 & $1 \cdot 05$ & 0.92 & $1 \cdot 14$ & 1.06 & $1 \cdot 31$ & $1 \cdot 22$ & $0 \cdot 87^{\circ}$ & $0 \cdot 86$ & 0.72 \\
\hline High density lipoprotein cholesterol (HDL) ( $\mathrm{mmol} / \mathrm{l})$ & -.04 & -.02 & -.02 & $-.06 \dagger$ & $-.08 t$ & -.04 & $-.06 \dagger$ & .01 & .03 & -.01 & .05 & -.07 \\
\hline Triglyceride $(\mathrm{mmol} / \mathrm{l})$ & .09 & .01 & $-\cdot 01$ & $.03^{\circ}$ & .03 & $\cdot 13$ & $\cdot 07^{*}$ & $.09+$ & $\cdot 11+$ & .06 & $\cdot 14 \dagger$ & $\cdot 13$ \\
\hline Low density lipoprotein cholesterol $(\mathrm{mmol} / \mathrm{l})$ & .06 & -.09 & $-\cdot 10$ & $\cdot 08$ & $-\cdot 04$ & $-\cdot 10$ & -.03 & -.04 & -.01 & $-.21 \ddagger$ & $-.23^{*}$ & $-\cdot 12$ \\
\hline TC/HDL & $\cdot 31 \dagger$ & .03 & .04 & $-25^{*}$ & .25 & $\cdot 21$ & $\cdot 11$ & -.00 & -.03 & -.04 & $-\cdot 19$ & .06 \\
\hline Height $(\mathrm{cm})$ & $0 \cdot 4$ & $0 \cdot 1$ & $0 \cdot 4$ & $0 \cdot 8^{*}$ & $0 \cdot 8$ & $0 \cdot 3$ & $0 \cdot 8^{*}$ & $0 \cdot 4$ & $-0 \cdot 3$ & $1 \cdot 1 \neq$ & $1 \cdot 0^{*}$ & $0 \cdot 2$ \\
\hline Weight (kg) & $0 \cdot 6$ & $0 \cdot 3$ & $1.5^{*}$ & $2 \cdot 5 \ddagger$ & $2 \cdot 7 t$ & $2 \cdot 5 *$ & $3 \cdot 3 \ddagger$ & $2 \cdot 4 \dagger$ & $2 \cdot 2 \dagger$ & $4 \cdot 4 \neq$ & $2 \cdot 3^{*}$ & $2 \cdot \overrightarrow{1}$ \\
\hline $\mathrm{BMI}\left(\mathrm{kg} / \mathrm{m}^{2}\right)$ & $0 \cdot 3$ & $0 \cdot 0$ & $0 \cdot 3$ & $0.6 \dagger$ & $0 \cdot 6$ & 0.6 & $1 \cdot 1 \neq$ & $0.9+$ & $1.0+$ & $1.4 \ddagger$ & 0.6 & $0 \cdot 8$ \\
\hline Overweight/obese & $1 \cdot 16$ & $1 \cdot 14$ & $1 \cdot 28$ & $1 \cdot 23$ & $1 \cdot 46^{*}$ & $1 \cdot 28$ & $1 \cdot 77 \ddagger$ & $1.73+$ & $1.95 t$ & $1 \cdot 68 \ddagger$ & $1 \cdot 34$ & $1 \cdot 23$ \\
\hline $\begin{array}{l}\text { Obese } \\
\text { Behavioural risk factors }\end{array}$ & 1.65 & 1.08 & 0.92 & $1 \cdot 31$ & $1 \cdot 28$ & $1 \cdot 39$ & $1.57^{*}$ & 1.66 & $5 \cdot 83 t$ & $1 \cdot 76 \ddagger$ & 1.56 & 1.85 \\
\hline Do not add salt & $1.97 \dagger \neq$ & $\ddagger 2.17 \ddagger$ & $2 \cdot 78 \ddagger$ & $1.73 \ddagger$ & $1.91 \ddagger$ & $2 \cdot 13 \ddagger$ & $1.87 \ddagger$ & $1 \cdot 56 \neq$ & $2 \cdot 79 \neq$ & $1 \cdot 57 \ddagger$ & $1.43^{*}$ & $1 \cdot 70+$ \\
\hline Do not eat fat & 1.06 & $1 \cdot 38^{*}$ & $1.44 \dagger$ & $1.37 \dagger$ & 1.05 & $1 \cdot 87 \ddagger$ & $1.46 \neq$ & $1.87 \ddagger$ & $2 \cdot 36 \ddagger$ & $1.60 \ddagger$ & $1.94 \ddagger$ & $1.65^{*}$ \\
\hline Current smoker & $0.55 \dagger \neq$ & $\ddagger 0.81$ & $0.64 \dagger$ & $0.58 \ddagger$ & $0.64 *$ & $0.48 t$ & 0.89 & $0.76^{\top}$ & $0.63^{*}$ & $0.83^{\circ}$ & $0.51 \neq$ & $0.52^{*}$ \\
\hline Light alcohol & $1.45^{* \top}$ & $1 \cdot 12$ & $1 \cdot 00$ & 1.09 & 1.03 & 0.78 & 0.79 & $0.51 \neq$ & 0.83 & $0.69 \ddagger$ & 0.97 & $1 \cdot 53$ \\
\hline Mod/heavy alcohol & $0 \cdot 42 \ddagger$ & $0.52 \dagger$ & $0.52 *$ & $0.55 \ddagger$ & $0 \cdot 70$ & 0.76 & $0.48^{*}$ & 0.69 & 0.52 & 0.89 & 0.88 & $0.27 \dagger$ \\
\hline Aerobic exercise & 1.00 & 1.04 & 0.79 & 1.25 & 0.79 & 1.40 & $0 \cdot 79$ & $1 \cdot 69^{*}$ & 0.97 & 1.07 & $0 \cdot 39^{*}$ & 0.71 \\
\hline Vigorous exercise & 1.02 & 1.00 & 1.00 & $1 \cdot 23$ & 1.01 & 0.96 & $1 \cdot 15$ & 0.89 & $0 \cdot 78^{*}$ & $1 \cdot 37^{*}$ & 0.97 & $1 \cdot 38$ \\
\hline Walking & $1 \cdot 38 \dagger$ & $1 \cdot 14$ & $1 \cdot 24^{*}$ & $1.43 \ddagger$ & $1 \cdot 13$ & $1 \cdot 23$ & $1 \cdot 22$ & 1.09 & $1.48 \dagger$ & $1 \cdot 25^{*}$ & $1 \cdot 21$ & 1.31 \\
\hline $\begin{array}{l}\text { No exercise } \\
\text { Multiple risk factors }\end{array}$ & Multiple risk factors & 1.06 & $0 \cdot 87$ & $0.67 \ddagger$ & $0 \cdot 89$ & 0.92 & $0 \cdot 85$ & $1 \cdot 19$ & 0.93 & $0.83^{*}$ & 0.89 & $0 \cdot 80$ \\
\hline $2-3$ classic & $0.67^{*}$ & $0 \cdot 71$ & $0.52^{*}$ & $0.71^{*}$ & $0.54 \dagger$ & $0 \cdot 54^{*}$ & 0.62 & $1 \cdot 14$ & 1.00 & $0.59 \ddagger$ & $0.43 \ddagger$ & 0.51 \\
\hline 2-4 biomedical & $1 \cdot 21$ & 1.30 & 0.73 & $1 \cdot 17$ & 0.85 & 0.72 & $1 \cdot 56^{*}$ & 3.04 & $3.06^{*}$ & 0.8 & $0.58^{*}$ & 0.68 \\
\hline $2-4$ behavioural & $0.55 \ddagger$ & $0.55 \ddagger$ & $0.43 \ddagger$ & $0.58 \ddagger$ & $0.49 \ddagger$ & $0.59^{*}$ & $0.70 \dagger$ & $0.73^{*}$ & $0.52 t$ & $0.67 \ddagger$ & $0.59 \dagger$ & $0.36 \dagger$ \\
\hline
\end{tabular}

${ }^{2}$ Differences in level (1989-80) or odds ratios $(1989 / 1980)$ by educational attainment adjusted for age and survey design parameters. ${ }^{*} \mathrm{p}<0 \cdot 05 . \dagger \mathrm{p}<0 \cdot 01, \ddagger \mathrm{p}<0 \cdot 001$.

Table 3 Inequalities ${ }^{\mathrm{a}}$ in risk factors in relation to survey year, sex, and age group

\begin{tabular}{|c|c|c|c|c|c|c|c|c|c|c|c|c|}
\hline \multirow[b]{2}{*}{ Variable } & \multicolumn{3}{|c|}{ Men 25-44y } & \multicolumn{3}{|c|}{ Men 45-64y } & \multicolumn{3}{|c|}{ Women $25-44 y$} & \multicolumn{3}{|c|}{ Women 45-64y } \\
\hline & 1980 & 1983 & 1989 & 1980 & 1983 & 1989 & 1980 & 1983 & 1989 & 1980 & 1983 & 1989 \\
\hline \multicolumn{13}{|l|}{ Biomedical risk factors } \\
\hline Systolic blood pressure $(\mathrm{mmHg})$ & $3 \cdot 6 \ddagger$ & $3 \cdot 6 \ddagger$ & $1 \cdot 0$ & 1.9 & $8 \cdot 4 \ddagger$ & 1.5 & $2 \cdot 2^{*}$ & $2 \cdot 1 \dagger$ & $1.9^{*}$ & $2 \cdot 5$ & $7 \cdot 2 \ddagger$ & $3 \cdot 3^{*}$ \\
\hline Diastolic blood pressure $(\mathrm{mmHg})$ & $1 \cdot 4^{*}$ & $2 \cdot 1 \ddagger$ & -0.0 & 0.5 & $4 \cdot 0 \ddagger$ & $0 \cdot 3$ & $1 \cdot 3^{*}$ & $1 \cdot 2^{*}$ & $1 \cdot 2^{*}$ & $1 \cdot 3$ & $2 \cdot 7 \ddagger$ & $0 \cdot 2$ \\
\hline Hypertension & 1.43 & $1.82 \dagger$ & 0.95 & 0.89 & $2 \cdot 00 \ddagger$ & $1.65 t$ & 1.47 & $1 \cdot 84^{*}$ & $2 \cdot 42^{*}$ & $1 \cdot 36$ & $1.55^{*}$ & $1.82 \dagger$ \\
\hline Total cholesterol (TC) $(\mathrm{mmol} / \mathrm{l})$ & -.02 & -.02 & $15^{*}$ & -.07 & .04 & .04 & $\cdot 19 t$ & .09 & .09 & $\cdot 18$ & .05 & $\cdot 14$ \\
\hline Raised TC & 0.75 & 0.95 & $1 \cdot 30$ & 0.89 & $1 \cdot 02$ & $0 \cdot 82$ & 1.45 & 1.58 & $1 \cdot 26$ & $1 \cdot 05$ & $1 \cdot 12$ & $1 \cdot 27$ \\
\hline High density lipoprotein cholesterol (HDL) $(\mathrm{mmol} / \mathrm{l})$ & .03 & $-0.4^{*}$ & .01 & .03 & -.02 & .01 & -.04 & $-\cdot 10 \ddagger$ & $\xi-\cdot 13 \ddagger$ & $-.09^{*}$ & $-.09+$ & $t-.03$ \\
\hline Triglycerides $(\mathrm{mmol} / \mathrm{l})$ & .09 & $\cdot 10$ & $\cdot 19 \dagger$ & $\cdot 10$ & $\cdot 14^{*}$ & $\cdot 00$ & $\cdot 11 t$ & $\cdot 14 \ddagger$ & $.08 t$ & $\cdot 22 \ddagger$ & $\cdot 16 \dagger$ & $\cdot 15 \dagger$ \\
\hline Low density lipoprotein cholesterol $(\mathrm{mmol} / \mathrm{l})$ & -.09 & -.04 & .07 & $-\cdot 13$ & .01 & .06 & $\cdot 19+$ & $-14 \dagger$ & $\cdot 18+$ & $\cdot 20^{*}$ & .05 & $\cdot 10^{\circ}$ \\
\hline TC/HDL & -.08 & $\cdot 18$ & $\cdot 19$ & -.04 & .09 & .00 & $\cdot 27 \dagger$ & $.35 \ddagger$ & $.41 \ddagger$ & $\cdot 34 \dagger$ & $\cdot 31^{*}$ & .24 \\
\hline Height $(\mathrm{cm})$ & $-2 \cdot 6 \ddagger$ & $-2 \cdot 8 \ddagger$ & $-2 \cdot 6 \ddagger$ & $-3 \cdot 6 \ddagger$ & $-3.4 \ddagger$ & $-3 \cdot 1 \ddagger$ & $-2 \cdot 5 \ddagger$ & $-1.6 \ddagger$ & $-1 \cdot 4 \ddagger$ & $-2 \cdot 4 \ddagger$ & $-2 \cdot 0 \ddagger$ & $-1.5 \dagger$ \\
\hline Weight (kg) & 0.5 & -0.3 & -0.3 & -1.6 & $-0 \cdot 7$ & -1.6 & $1 \cdot 3$ & $2 \cdot 4 \ddagger$ & $2 \cdot 4 \ddagger$ & -0.2 & $3 \cdot 2 \ddagger$ & $2 \cdot 1^{*}$ \\
\hline BMI $\left(\mathrm{kg} / \mathrm{m}^{2}\right)$ & $0.7 \dagger$ & $0 \cdot 7 \ddagger$ & $0.6 t$ & 0.4 & $0 \cdot 7 \dagger$ & 0.5 & $1 \cdot 2 \ddagger$ & $1.4 \ddagger$ & $1 \cdot 4$ & 0.6 & $1.9 \ddagger$ & $1 \cdot 3 \ddagger$ \\
\hline Overweight/obese & $1.67 \ddagger$ & $\neq 1.35^{*}$ & $1.51 \neq$ & $1 \cdot 27$ & $1.56 \ddagger$ & $1 \cdot 23$ & $2 \cdot 19 \ddagger$ & $1.92 \ddagger$ & $1.99 \ddagger$ & $1 \cdot 21$ & $1.91 \neq$ & $1.66 \dagger$ \\
\hline Obese & 0.94 & $2 \cdot 04 \dagger$ & $1 \cdot 68^{*}$ & $1 \cdot 74$ & $1.99 t$ & $1.63^{*}$ & $7 \cdot 26 \ddagger$ & $2 \cdot 32 \ddagger$ & $1.95 t$ & $1 \cdot 78$ & $2.36 \dagger$ & $1.69^{*}$ \\
\hline \multicolumn{13}{|l|}{ Behavioural risk factors } \\
\hline Do not add salt & na & $0.43 \ddagger$ & $0.30 \ddagger$ & na & $0.61 \ddagger$ & $0.50 \ddagger$ & na & $0.71 t$ & $0 \cdot 48 \ddagger$ & na & $0 \cdot 70^{*}$ & $0.64 \dagger$ \\
\hline Do not eat fat & $0 \cdot 84$ & na & $0.62 \ddagger$ & $1 \cdot 12$ & na & 0.82 & $1 \cdot 13$ & na & 0. & 1.00 & & 0.98 \\
\hline Current smoker & $2 \cdot 75 \ddagger$ & $2 \cdot 78 \ddagger$ & $2.38 \ddagger$ & $2 \cdot 12 \ddagger$ & $2 \cdot 29 \ddagger$ & $2 \cdot 56 \ddagger$ & $1.97 \ddagger$ & $1.97 \ddagger$ & F $2 \cdot 80 \ddagger$ & 0.88 & 1.09 & 1.40 \\
\hline Light alcohol & $0.41 \ddagger$ & $0.45 \ddagger$ & $0.60 t$ & $0.56 t$ & $0.53 \ddagger$ & 0.78 & $0.61 \dagger$ & $0.48 \ddagger$ & & $1 \cdot 00$ & $0.47 \ddagger$ & $0.45 \ddagger$ \\
\hline Mod/heavy alcohol & $3 \cdot 28 \ddagger$ & $2.03 \ddagger$ & $2 \cdot 67$ & $1 \cdot 87^{*}$ & $2.94 \ddagger$ & $1 \cdot 34$ & 0.78 & 0.69 & 0.72 & $0.43 t$ & 1.47 & 1.45 \\
\hline Aerobic exercise & na & $0.45 \ddagger$ & $0.57 \dagger$ & na & $0.43 \ddagger$ & $0.39 \ddagger$ & na & $0.51 \dagger$ & $0.41 \ddagger$ & na & $0.22 \ddagger$ & $0.34 \dagger$ \\
\hline us exercise & na & $0.39 \neq$ & $0.40 \ddagger$ & na & $0.33 \ddagger$ & $0.43 \ddagger$ & na & $0.40 \neq$ & & na & $0.49 \ddagger$ & $0.49 \ddagger$ \\
\hline Walking & na & $0.54 \ddagger$ & $0.60 \ddagger$ & na & $0.46 \ddagger$ & $0.54 \ddagger$ & na & $0.51 \ddagger$ & $0.42 \ddagger$ & na & $0.50 \neq$ & $0.47 \ddagger$ \\
\hline No exercise & na & $3 \cdot 31 \ddagger$ & F $\quad 3.03 \ddagger$ & na & $3.56 \neq$ & $2 \cdot 60 \ddagger$ & na & $2 \cdot 70 \ddagger$ & $2 \cdot 46 \ddagger$ & na & $2 \cdot 39 \ddagger$ & $2 \cdot 46 \ddagger$ \\
\hline \multicolumn{13}{|l|}{ Multiple risk factors } \\
\hline 2-3 Classic & $1.80 \dagger$ & $2 \cdot 06 \ddagger$ & $2 \cdot 35 \ddagger$ & $1 \cdot 00$ & $2 \cdot 57 \ddagger$ & $1 \cdot 32$ & 1.95 & $2 \cdot 87^{*}$ & 1. & $1 \cdot 10$ & 1.69 & $1 \cdot 26$ \\
\hline 2-4 Biomedical & 0.85 & $1.86 \dagger$ & $1 \cdot 40$ & 0.98 & $1.74 \dagger$ & $1.58^{*}$ & $4 \cdot 14 \dagger$ & $3.03 \ddagger$ & $2 \cdot 11 t$ & 1.59 & $2.01 \dagger$ & $1.96 \dagger$ \\
\hline 2-4 Behavioural & & $4 \cdot 08 \ddagger$ & $5 \cdot 21 \ddagger$ & na & $3.48 \ddagger$ & $3.44 \ddagger$ & na & $2 \cdot 48 \ddagger$ & $3 \cdot 31 \neq$ & na & $1.75 \dagger$ & $3 \cdot 26 \ddagger$ \\
\hline
\end{tabular}

a Differences in level (low-high educational attainment) or odds ratios (low/high educational attainment) by survey year adjusted for age and survey design parameters. $\mathrm{na}=$ not available, question not asked. ${ }^{*} \mathrm{p}<0 \cdot 05, \mathrm{p} \mathrm{p}<0 \cdot 01, \neq \mathrm{p}<0 \cdot 001$.

more strongly associated in women than men (similarly for LDL). LDL levels decreased significantly among older women with low or medium education. TC/HDL increased significantly among men of low education. Women exhibited socioeconomic gradients in TC/HDL which favoured high educational attainment and there were no statistically significant trends over time.

Younger men of high educational attainment were $2.7 \mathrm{~cm}$ taller on average than men of the same age of low educational attainment. The differential for older men was $3.3 \mathrm{~cm}$, for younger women $1.9 \mathrm{~cm}$, and for older women $2.0 \mathrm{~cm}$ (derived from table 3 ). The average weight of women increased from between $2 \cdot 1$ and $4.4 \mathrm{~kg}$ over the study period depending on age and educational attainment, with greater increases among women of low educational attainment (table 2). Older men experienced increases of around $2.5 \mathrm{~kg}$ regardless of educational attainment. BMI was greatest among women of low educational attainment, who also showed the greatest increases over time. The greatest increases in the proportion overweight or obese were experienced by younger women independent of educational attainment, and by older women of lower education (table 2, fig 1).

With regard to dietary behaviour, "not adding salt to food" became more common at each level of educational attainment (fig 2). This 

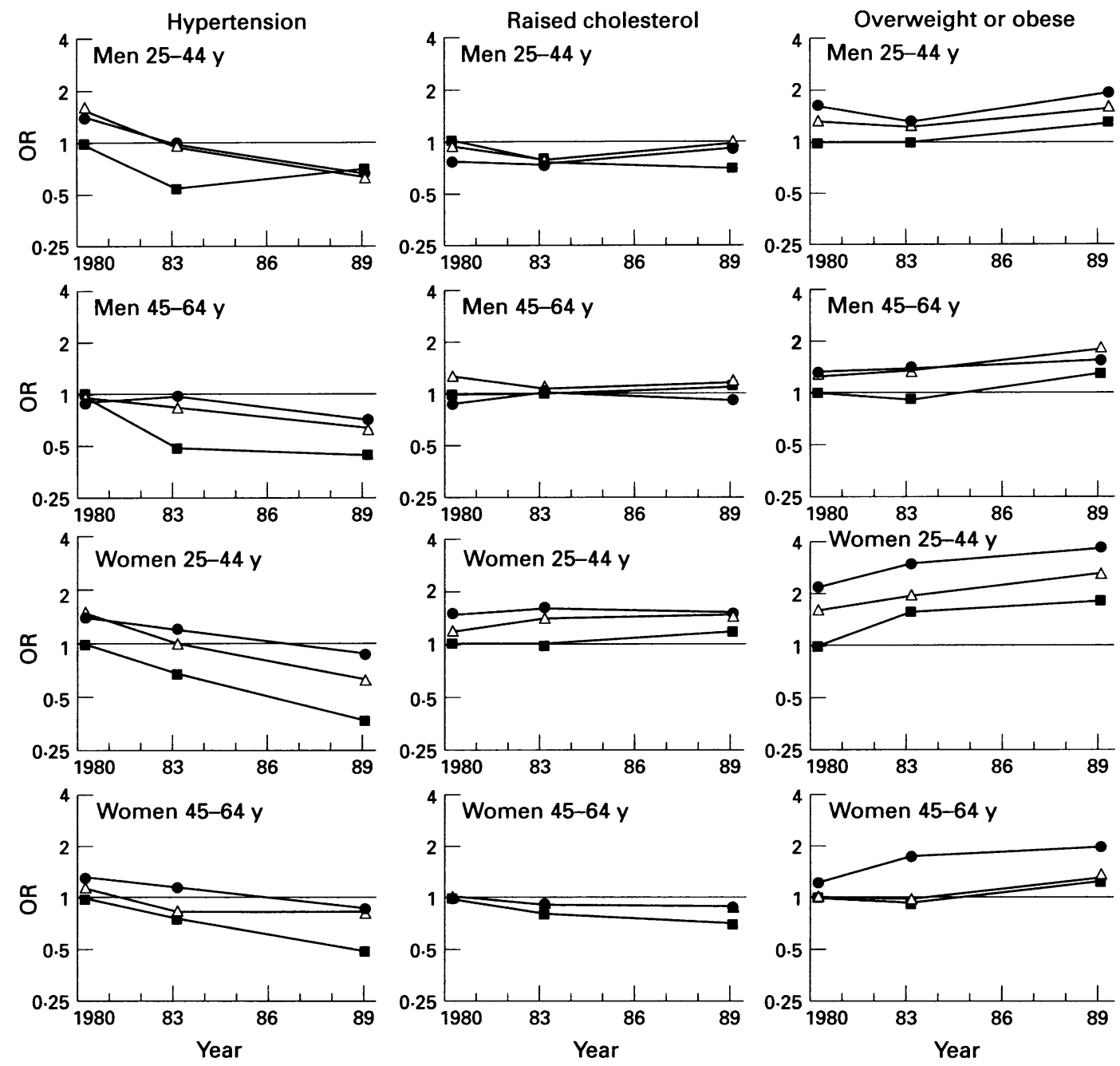

Educational attainment - Low $\Delta$ Medium - High

Figure 1 Selected biomedical risk factors - odds ratios (OR) in relation to educational attainment, sex, age group, and year. The reference group is high education in $1980(O R=1)$.

was observed for each age-sex group, although the data suggest greater improvements among younger, tertiary educated men and women (table 2). Significant gradients existed with educational attainment for each age-sex group and were maintained over time (table 3). Education gradients were not as marked for "not eating the fat on meat", and were statistically significant only for younger men and women in 1989 (table 3). All time trends were statistically significant with the exception of younger men of low educational attainment and older men of medium educational attainment.

In each age-sex group, smoking prevalence declined significantly among those of high educational attainment (table 2). There were declines in the other educational groups but, with the exception of older women, the clear differentials in favour of high educational attainment persisted (table 3). Trends were least and non-significant among younger and older women of low educational attainment (table 2, fig 2).

Among younger men, the prevalence of moderate or heavy drinking declined at each level of educational attainment, although the socio- economic gradient persisted. The high prevalence of moderate or heavy drinking reported in 1980 by older women with high educational attainment had significantly declined by 1983 and was maintained in 1989. For each age-sex group, light alcohol drinking tended to be more popular among those of high educational attainment.

All forms of leisure-time exercise were strongly associated with educational attainment (table 3). Those of high educational attainment were more likely to participate than those of low attainment (fig 2). There was little change over time for men in participation rates for aerobic exercise or vigorous exercise (table 2). For women, the data suggest changes in participation rates for aerobic exercise for those of medium educational attainment, increasing participation for younger women and decreasing for older women. Each age-sex group reported increased participation in walking for recreation or exercise at each level of educational attainment, although increases were less for those of medium educational attainment. Using "no exercise in the past 2 weeks" as a summary measure shows that the 
Do not add salt
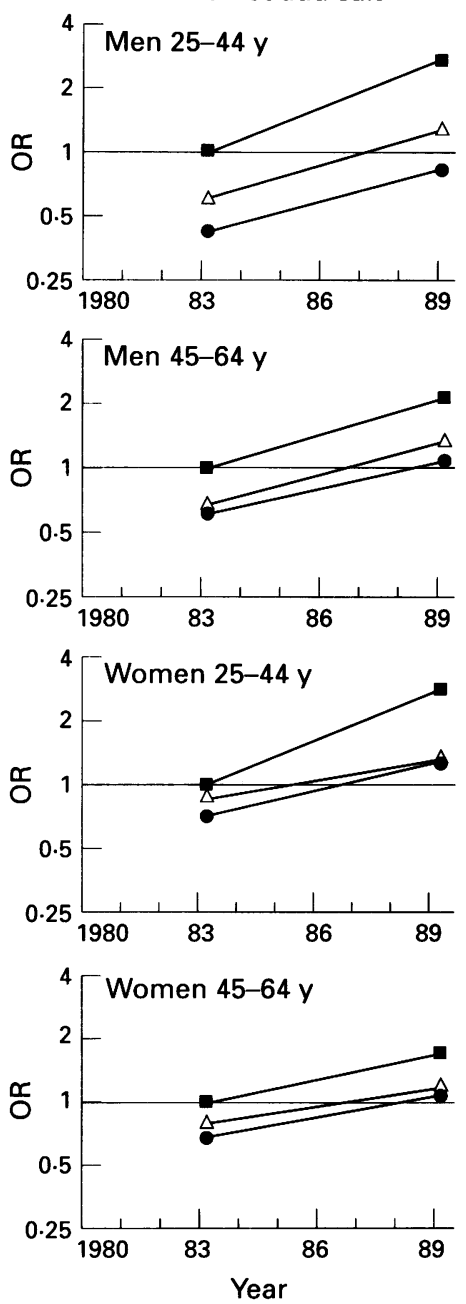

Current smokers

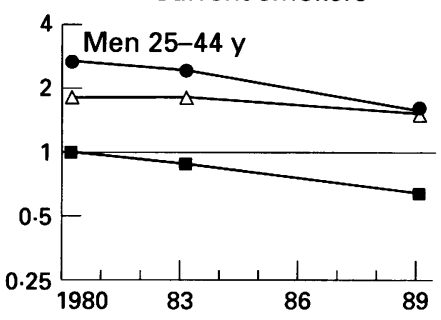

4 Men 45-64 y
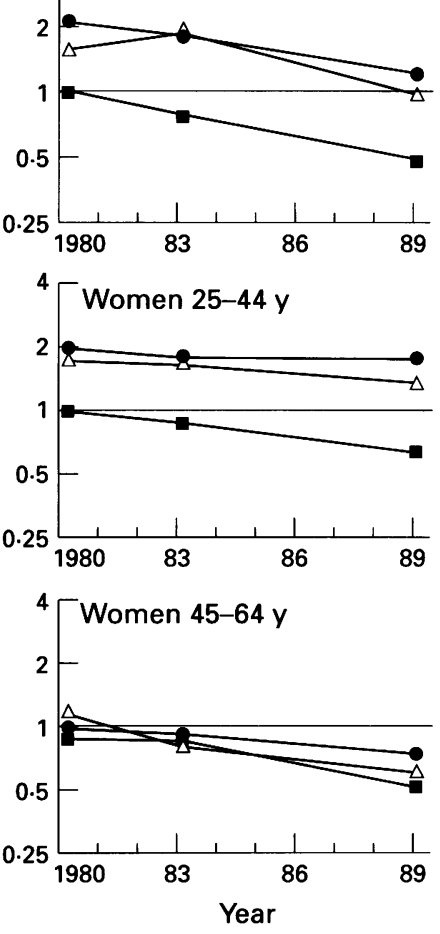

Educational attainment

- Low $\Delta$ Medium - High
No exercise
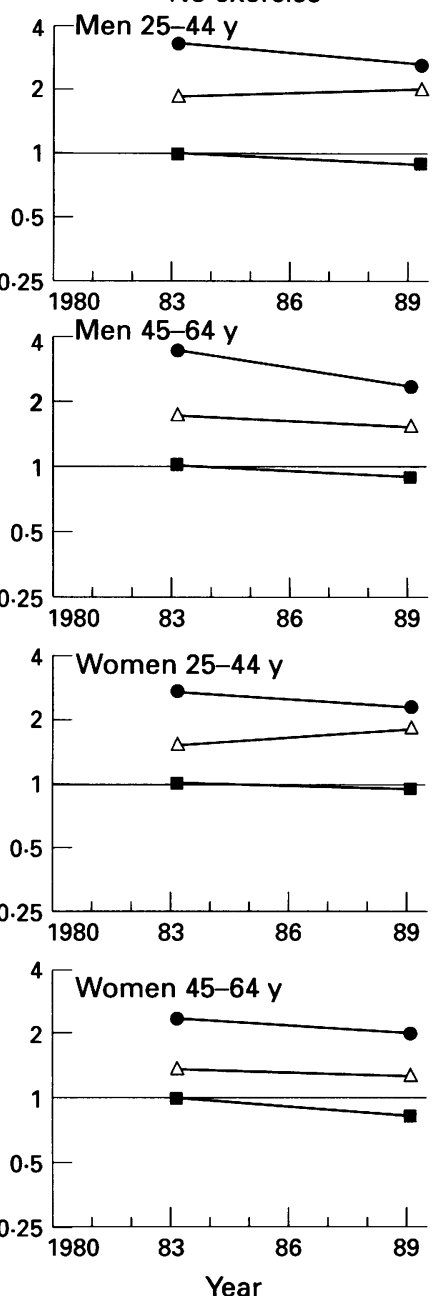

Figure 2 Selected behavioural risk factors - odds ratios (OR) in relatin to educational attainment, sex, age group, and year. The reference group is high education in $1980(O R=1)$.
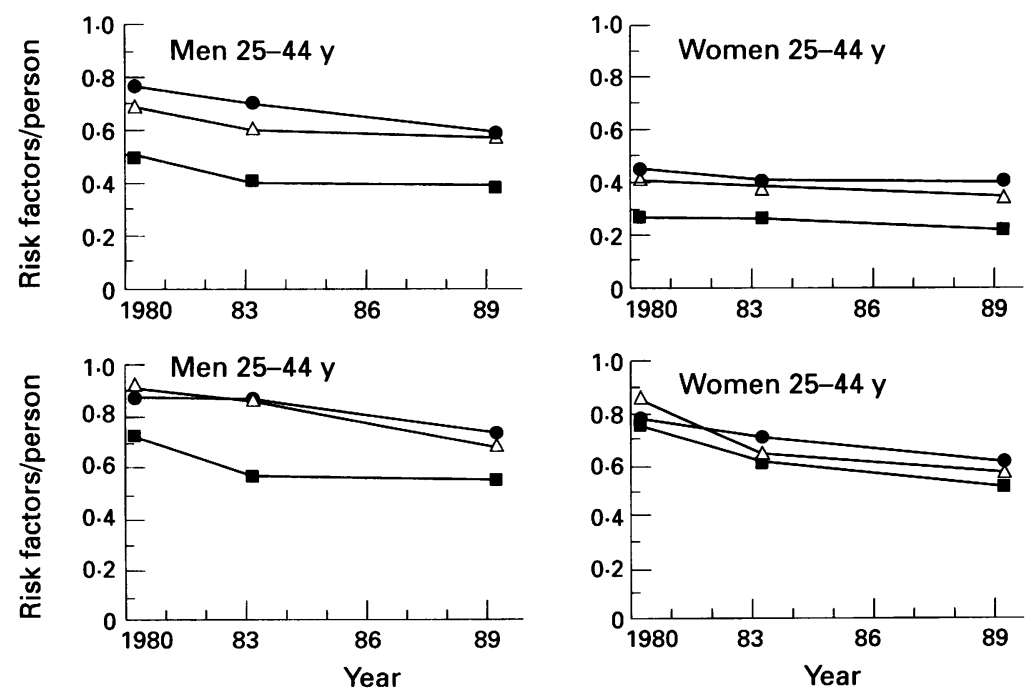

Educational attainment - Low $\triangle$ Medium - High

Figure 3 Classic risk factors (high diastolic blood pressure, high total cholesterol concentration, and cigarette smoking) - average number per person in relation to educational attainment, sex, age group, and year. socioeconomic gradient observed for physical inactivity during leisure time persisted over time, with the greatest improvement shown by older men of low educational attainment.

The prevalence of multiple behavioural risk factors displayed strong (inverse) associations with educational attainment for each age-sex group, resulting in prevalence ORs around 2 to 5 for low compared with high educational attainment (table 3). Nevertheless, each educational group experienced an improvement in their health risk behaviour profile over the study period (table 2).

An inverse association with educational attainment was also consistently observed for the prevalence of multiple biomedical risk factors among younger and older women. The results suggest a worsening of the biomedical risk factor profile for younger women irrespective of educational attainment and little improvement in the older age, sex groups.

Only among young men was the simultaneous occurrence of two or more of the three classical risk factors for coronary heart disease inversely associated with educational 
attainment at each survey (table 3). Generally, statistically significant decreases in the prevalence of multiple risk factors were observed at each educational level (table 2), except for young women where prevalences were already less than $6 \%$.

\section{Discussion}

The risk factor prevalence study provides a unique opportunity to examine trends in socioeconomic differentials in cardiovascular risk factors in a representative sample of urban Australians. Few studies have reported trends in risk factor inequalities and those that have tend to be of single local or regional communities. ${ }^{243233}$ The present data analysis is based on nationwide probability sample surveys of urban living Australian adults aged 25-64 with an average sample size of 6400 respondents. The results improve our understanding of cardiovascular disease aetiology in society, and help in formulating policy and determining health promotion activities.

\section{EDUCATIONAL ATTAINMENT}

Of the three most commonly used indicators of socioeconomic status - educational attainment, occupation, and income - education has been found to be more strongly associated with disease, ${ }^{2223}$ including coronary heart disease. ${ }^{26}$ The most plausible mechanism by which education protects against disease is a favourable influence on lifestyle, problem solving abilities, and values. ${ }^{22} 23$

The level of educational attainment of Australians generally has been increasing steadily in recent years ${ }^{34}$ and this is clearly reflected in the survey samples (table 1). Also clearly shown is that younger survey participants are more likely to have experienced post-compulsory education (after the age of 15 years) than those in the older age group. Conducting the analysis separately for two broad age groups allows for an age-specific effect, and recognises that postcompulsory education may have a different meaning for different age groups. The trend towards increasing homogeneity of educational attainment may affect the future usefulness of educational attainment as a marker of socioeconomic status.

\section{BLOOD PRESSURE}

Mean blood pressure tended to be higher among those with lower educational attainment but the difference was not statistically significant at all points in time. Some recent studies have reported a negative association between blood pressure and level of education in women but not in men..$^{223035}$ Others have found the relationship in both men and women, ${ }^{26}$ and others have found no such relationship. ${ }^{30}$ This may reflect the dynamic influence of other related factors such as diet, treatment for hypertension, and smoking behaviour. ${ }^{26}$ The results of this study show that the association can fluctuate over time within the same population subgroup.
The cause(s) of the falls in mean blood pressure over time remains unknown. The sizes of the decreases, which occurred for each level of educational attainment in each age-sex group, are too great to be explained by measurement error. ${ }^{36}$ Blood pressure was highly correlated with BMI and to a lesser extent with smoking status. Controlling for these variables did not explain the falls; in fact many of the decreases were magnified. The estimates of trends and inequalities were essentially unaffected by excluding from the analysis subjects on antihypertensive medication.

\section{LIPIDS}

The results suggest an inverse association between average TC and the level of education among women but not among men, a finding reported elsewhere. ${ }^{22}$

Most published reports show inconsistent results, however, both in terms of the direction and strength of the association, ${ }^{262730}$ which may be related to variation in the level of public concern and individual awareness in the communities in which the studies were conducted. The overall lipid picture in this study is one that favours women of high educational attainment who, in addition to lower TC concentrations, also had higher average concentrations of HDL, lower triglyceride, lower LDL, and lower TC/ HDL

The dietary messages of the 1980 s, to reduce the intake of saturated fat, had little effect on the lipid profile of any population subgroup. Younger women experienced no change in average TC at any level of educational attainment. Average TC concentrations decreased in older women, especially those of low educational attainment. Among men, there were few changes in the lipid profile of any educational group, the most notable trends being statistically significant increases in TC/HDL ratios among men of low educational attainment.

\section{HEIGHT}

These data show, for each survey and age-sex group, the well known association between adult height and socioeconomic status. Reasons for the association are uncertain but adult height has been suggested as a good indicator of net nutritional status and average health status. ${ }^{37}$ The association may therefore reflect differences in nutrition and health during early childhood, and the statistically significant increases in the average height of men (older) and women of lower educational attainment may reflect improvements in conditions during childhood in these socioeconomic groups. Height is known to be a predictor of all cause mortality and coronary heart disease mortality, at least in middle aged men. ${ }^{19}$

\section{BM}

The inverse relationship between BMI and educational attainment was also repeated at each survey. The relationhip was strongest for women age $25-44$, for whom ratios close to 2 were observed in each survey for the relative 
odds of being overweight or obese (table 3). The prevalence of women in this age group who were overweight or obese in 1989 was still less than that in the other age-sex groups despite the significant increases which occurred at each level of educational attainment. Excess body weight is known to be associated with increased mortality, and there is some evidence that change in body weight may be similarly associated. $^{38}$

BMI was inversely related to smoking status, especially among older men and women and to a lesser extent among younger men, but not in younger women. Consequently, smoking status could not explain the higher mean BMIs in those of low educational attainment since smoking was more prevalent in that strata. As expected, when smoking status was added to the model the socioeconomic differentials were slightly increased. It is possible to hypothesise, however, that the decreases in the prevalence of smoking over time could have contributed to the increases in mean BMI. In fact, controlling for smoking status did slightly moderate the increases in mean BMI among older men and women, but did not completely explain them, consistent with an independent analysis. $^{39}$

\section{DIETARY BEHAVIOUR}

The questions on discretionary use of salt and propensity to eat the fat on meat are useful for monitoring behaviour and awareness of health messages. They are not intended as measures of sodium or saturated fat intake. The advice to avoid salt was adopted across the spectrum of educational attainment but with no suggestion that the socioeconomic gradient, which favoured the more highly educated, was diminishing. The results suggest the emergence of educational differentials among younger men and women in respect of attitudes towards eating the fat on meat.

\section{SMOKING}

In both younger and older men, the prevalence of smoking was consistently less among the higher educated and all groups experienced parallel declines in smoking prevalence. This suggests that men of all educational levels responded positively to the antismoking messages of the 1980s while maintaining the relative differences between groups. The picture is different among both younger and older women, for whom the decline has been less among those in the low education group. That is, women of low educational attainment have been least likely to respond to past primary prevention activities and may be an appropriate target group for future health promotion programs. In contrast to the other age-sex groups, there as no evidence of a socioeconomic gradient for smoking prevalence among older women.

ALCOHOL

In 1980, the prevalence of moderate or heavy drinkers was highest among men of low edu- cational attainment, both younger and older men, and the significant reduction in this prevalence over the 1980 s is good news. The data suggest a negative association between educational attainment and moderate or heavy alcohol consumption in men, but a positive association in women, a finding consistent with other studies. ${ }^{3540}$ The sharp decrease in the prevalence of moderate or heavy drinkers among older women of high educational attainment is, perhaps, a good example that such associations are not immutable. The surveillance of light alcohol consumption is relevant to coronary risk given recent evidence that modest alcohol consumption is linked to lower cardiovascular risk. ${ }^{4142}$ In this study the socioeconomic gradient invariably favours those with higher educational attainment.

\section{EXERCISE}

The lack of standardisation in the definition and assessment of physical activity in epidemiological research ${ }^{43}$ means that comparisons between surveys are uncommon. This present study used the same, self reported, twoweek recall questionnaire technique to measure leisure time activity in two surveys six years apart, which means that, although comparison of absolute levels of exercise with other studies may be problematic, the data are suitable for the internal identification of exercise differentials, both socioeconomic and secular.

The strong positive association between educational attainment and physical activity during leisure time, in all subgroups and both surveys, is supported by similar findings from other studies. ${ }^{263035}$ The implications of this for coronary risk among women is clear, but not so for men. Other studies have found, among men but not women, that the lower levels of participation in physical activity during leisure time among those of lower educational attainment may be compensated for by increased physical activity at work..$^{3540}$ This could well apply in the present study, as the correlation between educational attainment and occupational status for men was highly significant $(\mathrm{r}=+0.54 ; \mathrm{p}<.0001)$; that is, men of lower educational attainment were more likely to be in manual occupations. Further, it is known that leisure time activity and exercise at work are both associated with reduced coronary risk, although their relative importance is unclear. ${ }^{44}$

Regarding trends over time, few were statistically significant at any level of activity. There seems to have been a general increase in walking as a form of exercise regardless of level of education, however, the data are self reported and there is no way of telling whether the trend reflects increased awareness or actuality.

\section{MULTIPLE RISK FACTORS}

All education groups responded positively to the health education messages of the 80 s and adopted healthier profiles of risk factor behaviours (table 2). Adults with lower education remained more likely to report an unhealthy lifestyle (table 3). Cigarette smoking and excess 
drinking were correlated at each level of educational attainment but especially among older women of high educational attainment. Among men, cigarette smoking was also correlated with lack of exercise and with increased discretionary use of salt.

The strong socioeconomic gradient for the prevalence of two or more risk behaviours within the one individual was consistent with the socioeconomic gradient for multiple biomedical risk factors (table 3). The gradient was observed also for the average number of classic risk factors per person (fig 3 ). With the exception of older women, the data suggested lower risk among the tertiary educated. Decreased risk of coronary events with increasing educational attainment is consistent with the known socioeconomic gradient in mortality for coronary heart disease. Overall, the results support the view that differentials in cardiovascular mortality may, at least in part, be ascribed to differences in biomedical and behavioural risk factors.

\section{RESPONSE BIAS}

Although the overall response rate of $75 \%$ was very high for surveys which include physical examination and venipuncture, it is sensible to consider the possible effects of reponse bias on the analysis. Other studies have found that people with an adverse risk factor profile are less likely to participate in surveys such as these. ${ }^{4546}$ If this were the case in our study, the inequalities observed would be underestimates of the true differentials, since risk factors are more prevalent in low socioeconomic groups. Overestimation of the association between educational attainment and risk factors would occur only if propensity to respond was associated with a better risk factor profile among the better educated and worse profile among the less educated, a phenomenon which has not been reported in other studies. There are no data available on the characteristics of nonrespondents in the present trend analysis but the remarkable consistency in overall response rates over time offers some assurance against biased trend estimates. Characteristics of nonrespondents have been found to be relatively stable over time. ${ }^{47}$

It is clear that Australians of different educational attainment have very different risk factor profiles. Socioeconomic inequalities are more pronounced for behavioural than biomedical risk factors, and for hypertension and body fatness that blood lipids. For both biomedical and behavioural risk factors, the strength of the associations varies with sex and age and inequalities are more common among women than men.

The lower socioeconomic group has improved its risk factor profile but its relative disadvantage compared with the higher socioeconomic group persists. This suggests that the health promotion activities in Australia have been effective in reaching the lower socioeconomic groups but that the challenge to reduce inequalities remains. The increase in weight is an unfavourable development shared by each socioeconomic group which, coupled with little reduction in smoking prevalence among women of low socioeconomic standing, might be expected to retard cardiovascular benefits in that population subgroup unless they are addressed.

Educational attainment may be a surrogate for other factors (for example, income which makes it easier to make health lifestyle choices) but it is also possible that exposure to higher formal education has a direct effect on risk factor levels and behaviours. The effect might be through the curriculum content or the intellectual training involved. Both might increase people's ability to understand and relate to health promotion exposure after formal education has ceased ${ }^{26}$ and improve health literacy. Regardless of the mechanism behind the association between education and risk factors, it is plausible that the steady increase in educational attainment in Australia has been an important factor in the general improvement in the nation's risk factor profile and in the decrease in mortality from coronary heart disease.

The author thanks the Risk Factor Prevalence Study Management Committee for permission to analyse data from the agement Committee for permission to analyse data from the
risk factor prevalence surveys, and Dr Erich Kliewer, National risk factor prevalence surveys, and Dr Erich Kliewer, National
Centre for Epidemiology and Population Health, Australian Centre for Epidemiology and Population Health, Australian
National University, Professor Susan Wilson, Centre for MathNational University, Professor Susan Wilson, Centre for Mathematical Applications, Australian National University, Dr Tony Worsely, Division of Human Nutrition, CSIRO, and Dr Paul
Magnus, National Heart Foundation of Australia, for their comments.

1 McMichael AJ, Hartshorne JM. Mortality risks in Australian men by occupational groups, 1968-1978. Med $\mathcal{f}$ Aust men by occup

2 Taylor R, Herrman H, Preston G. Occupation and mortality in Australia. Working age males, 1975-77. Melbourne: Health Commission of Victoria and the Department of Social and Preventive Medicine, Monash University, 1983.

3 Gibberd RW, Dobson AJ, Florey C duVe, Leeder SR. Differences and comparative declines in ischaemic heart disease mortality among subpopulations in Australia, 1969-1978. Int f Epidemiol 1984;13:25-31.

4 Dobson AJ, Gibberd RW, Leeder SR, O'Connell DL. Occupational differences in ischaemic heart disease mortality and risk factors in Australia. Am f Epidemiol 1985;122. and risk

5 McMichael AJ. Social class (as estimated by occupational prestige) and mortality in Australian males in the 1970s. Community Health Stud 1985;9:220-30.

6 Simons LA, Simons J, Magnus P, Bennett SA. Education level and coronary risk factors in Australians. Med $\mathcal{F}$ Aust 1986;145:446-50.

7 Siskind V, Copeman R, Najman JM. Socioeconomic status and mortality: A Brisbane area analysis. Community Health Stud 1987;11:15 23 .

8 Dobson A, Halpin S, Alexander H. Does the occupational structure of the Hunter region explain the high rates of ischaemic heart disease among its men? Aust 7 Public Health 1991;15:172-7.

9 Siskind V, Najman JM, Veitch C. Socioeconomic status and mortality revisited: an extension of the Brisbane area mortality revisited: an extension of the Brisba

10 National Health Strategy. Enough to make you sick. How income and envimnment affect health. Melbourne: National income and envirnment affect health. Melbourne:

11 Hardes GR, Dobson AJ, Lloyd DM, Leeder SR. Coronary heart disease mortality trends and related factors in Australia. Cardiology 1985;72:23-8.

12 Marmot MG, McDowall ME. Mortality decline and widening social inequalities. Lancet 1986;ii:274-6

13 Feldman JJ, Makuc DM, Kleinman JC, Cornoni-Huntley J. National trends in educational differentials in mortality. Am f Epidemiol 1989;129:919-33.

14 Marmot MG, Kogevinas M, Elston MA. Social/economic status and disease. Annu Rev Publ Health 1987;8:111-35.

15 Feinstein JS. The relationship between socioeconomic status and health: a review of the literature. Milbank $Q$ 1993:71: 279-322.

16 Black D. Inequalities in health: report of a working group. London: DHSS, HMSO, 1980.

17 Macintyre $S$. The patterning of health by social position in contemporary Britain: directions for sociological research. Soc Sci Med 1986;23:393-415.

18 Hart $N$. Inequalities in health. The individual versus the environment. $7 R$ Statist Soc $A$ 1986;149:228-46. 
19 Marmot MG, Shipley MJ, Rose G. Inequalities in deathspecific explanations of a general pattern? Lancet 1984;i: 1003-6.

20 Pocock SJ, Shaper AG, Cook DG, Phillips AN, Walker M. Social class differences in ischaemic heart disease in British men. Lancet 1987;ii:197-201.

21 Bennett $S$, Magnus $P$. Trends in cardiovascular risk factors in Australia. Results from the national heart foundation's risk factor prevalence study, 1980-1989. Med $\Im$ Aust 1994; risk factor preva

22 Winkleby MA, Jatulis DE, Frank E, Fortmann SP. Socioeconomic status and health: How education, income, and occupation contribute to risk factors for cadiovascular disease. Am $\mathcal{F}$ Public Health 1992;82:816-20.

23 Liberatos P, Link BG, Kelsey JL. The measurement of social class in epidemiology. Epidemiol Rev 1988;10:87-121

24 Winkleby MA, Fortmann SP, Barrett DC. Social class disaparities in risk factors for disease: Eight-year prevalence patterns by level of education. Prev Med 1990;19:1-12.

25 Holme I, Helgeland A, Hjermann I, Leren P, Lund-Larson PG. Four-year mortality by some socioeconomic indicators: the Oslo study. F Epidemiol Community Health 1980;34:48-52.

26 Jacobsen BK, Thelle DS. Risk factors for coronary heart disease and level of education. The Tromso heart study. Am 7 Epidemiol 1988;127:923-32.

27 Reynes J, Lasater TM, Feldman H, Assaf AR, Carleton RA. Education and risk factors for coronary heart disease: results from a New England community. Am $\mathcal{F}$ Prev Med 1993;9:365-71.

28 Risk Factor Prevalence Study Management Committee. Risk factor prevalence study: survey No 3 1989. Canberra: National Heart Foundation of Australia and Australian Institute of Health, 1990.

29 The Toronto Working Group on Cholesterol Policy. Asymptotic hypercholesterolemia: a clinical policy review. Chapter 2 . Serum cholesterol and other risk factors for coronary heart disease. $¥$ Clin Epidemiol 1990;43:1035-51.

30 Helmert U, Shea S, Herman B, Greiser E. Relationship of social class characteristics and risk factors for coronary heart disease in West Germany. Public Health 1990;104: 399-416.

31 National Health and Medical Research Council. Is there a safe level of daily consumption of alcohol for men and women? Recommendations regarding responsible drinking behaviour. Canberra: Australian Government Publishing Service, 1987.
32 Brannstrom I, Weinehall L, Persson LA, Wester PO, Wall $S$. Changing social pattern of risk factors for cardiovascula disease in a Swedish community intervention programme. Int 7 Epidemiol 1993;22:1026-37.

33 Leupker RV, Rosamond WD, Murphy R, Sprafka JM, Folsom AR, McGovern PG, Blackburn H. Socioeconomic status and coronary heart disease risk factors trends. The Minnesota heart survey. Circulation 1993;88:2172-9.

34 Australian Bureau of Statistics. Education. In: Australian social trends 1994. Canberra: ABS, 1994. Cat No 4102.0. 35 Garrison RJ, Gold RS, Wilson PWF, Kannel WB. Educational attainment and coronary heart disease risk: the Framingham offspring study. Prev Med 1993;22:54-64.

36 Bennett S. Blood pressure measurement error: its effect on cross-sectional and trend analyses. F Clin Epidemiol 1994 47:293-301.

37 Carr-Hill RA. Time trends in inequalities in health. $\mathcal{F}$ Biosoc Sci 1988;20:265-73.

38 Kushnar RF. Body weight and mortality. Nutr Rev 1993 51:127-36.

39 Boyle CA, Dobson AJ, Egger G, Magnus P. Can the increasing weight of Australians be explained by the decreasing prevalence of cigarette smoking? Int $\mathcal{f}$ Obesity creasing prevalenc

40 Woodward M, Shewry MC, Smith WCS, Tunstall-Pedoe $H$. Social status and coronary heart disease: results from he Scottish heart health study. Prev Med 1992;21:136-48. 41 Marmot M, Brunner E. Alcohol and cardiovascular disease: The status of the U shaped curve. BMf 1991;303:565-8. 42 Jackson R, Scragg R, Beaglehole R. Alcohol consumptio and risk of coronary heart disease. BMF 1991;303:211-6. 43 Lee C. Definition and assessment of physical activity. $A m$ f Public Health 1993;17:190-4.

44 Salonen JT, Slater JS, Tuomilehto J, Rauramaa R. Leisure time and occupational physical activity: Risk of death from ischaemic heart disease. Am $\mathcal{F}$ Epidemiol 1988;127:87-94.

45 Bergstrand R, Vedin A, Wilhelmsson C, Wilhelmsen L. Bia due to non-participation and heterogenous sub-groups in due to non-participation and heterogenous sub-gro

46 Criqui MH, Barrett-Connor E, Austin M. Differences between respondents and non-respondents in a populationtween respondents and non-respondents in a population108:367-72.

47 Sprafka JM, Burke GL, Folsom AR, Leupker RV, Blackburn $\mathrm{H}$. Continued decline in cardiovascular disease risk factors: results of Minnesota heart survey, 1980-1982 and 1985-1987. Am ₹ Epidemiol 1990;132:489-500. 